\title{
Tuberculin reactivity among health care workers at King Abdulaziz University Hospital, Saudi Arabia
}

E.A. Koshak ${ }^{1}$ and R.Z. Tawfeeq ${ }^{2}$

$$
\begin{aligned}
& \text { تفاعلية التوبر كلين بين العاملين الصحيين في جامعة الملك عبد العزيز في المملكة العربية السعودية }
\end{aligned}
$$

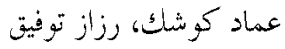

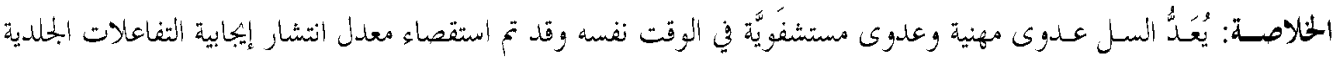

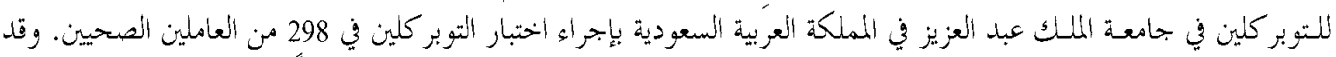

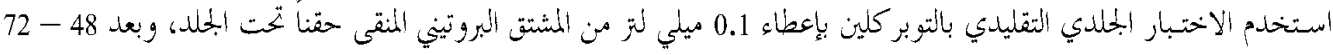

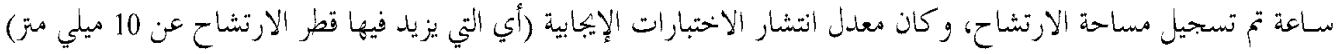

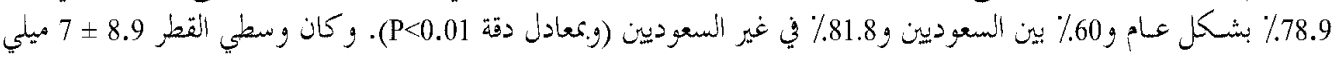

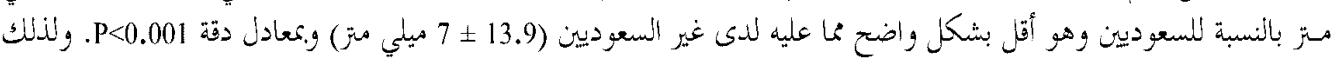

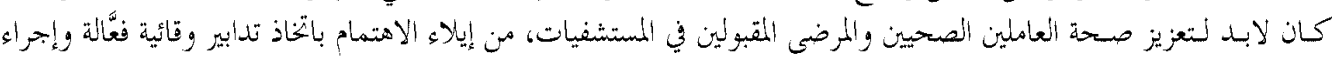

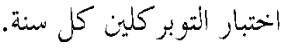

ABSTRACT Tuberculosis is both a nosocomial and an occupational infection. The prevalence of positive tuberculin skin reactions at King Abdulaziz University Hospital, Saudi Arabia, was investigated by testing 298 health care workers. Conventional tuberculin skin testing was performed using $0.1 \mathrm{~mL}$ of purified protein derivative injected intracutaneously. After 48-72 hours, induration size was recorded. The prevalence of positive tests (induration $\geq 10 \mathrm{~mm}$ ) was $78.9 \%$ overall, $60.0 \%$ for Saudi Arabians compared with $81.8 \%$ for non-Saudi Arabians $(P<0.01)$. The mean response size $(8.9 \pm 7.1 \mathrm{~mm})$ for Saudis was also significantly lower than for non-Saudis $(13.9 \pm 7.1 \mathrm{~mm}, P<0.001)$. To enhance the protection of both health care workers and hospitalized patients, effective preventive measures and annual tuberculin testing of health care workers should be considered.

La réactivité à la tuberculine chez les agents de soins de santé à l'Hôpital universitaire King Abdulaziz (Arabie saoudite)

RESUME La tuberculose est à la fois une infection nosocomiale et professionnelle. La prévalence des intradermo-réactions à la tuberculine positives à l'Hôpital universitaire King Abdulaziz en Arabie saoudite a été examinée en testant 298 agents de soins de santé. Le test cutané à la tuberculine conventionnel a été réalisé en utilisant $0,1 \mathrm{~mL}$ de tuberculine purifiée injectée par voie intradermique. Après $48-72$ heures, la taille de l'induration a été relevée. La prévalence des tests positifs (induration $\geq 10 \mathrm{~mm}$ ) était de $78,9 \%$ de manière générale, elle était de $60,0 \%$ pour les Saoudiens contre $81,8 \%$ pour les non-Saoudiens $(p<0,01)$. La taille moyenne de l'induration $(8,9 \pm 7 \mathrm{~mm})$ pour les Saoudiens était significativement moindre que pour les nonSaoudiens $(13,9 \pm 7 \mathrm{~mm}, p<0,001)$. Des mesures de prévention efficaces et un test à la tuberculine annuel pour les agents de soins de santé devraient être envisagés afin de renforcer la protection des agents de soins de santé et des patients hospitalisés.

${ }^{1}$ Department of Internal Medicine; ${ }^{2}$ Department of Family Medicine, King Abdulaziz University Hospital, Jeddah, Saudi Arabia.

Received: 25/10/01; accepted: 14/02/02

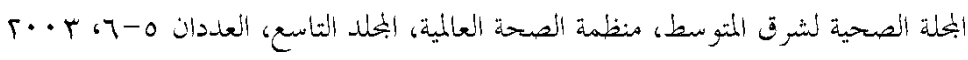




\section{Introduction}

Tuberculosis (TB) is a common communicable disease and may be considered both a nosocomial and an occupational infection [1,2]. In the United States of America (USA), the annual number of reported cases of TB increased by 14\% from 1985 to 1993 [3]. Consequently, concerns about this disease have increased and more attention is now being given to control measures. This has led to a decrease in the prevalence rate of TB to 7.4 cases per 100000 general USA population in 1997 [4]. Yet a number of groups, including health care workers, continue to show rising rates of TB infection [4,5].

Health care workers are exposed to a variety of infections, including TB, as they perform their job responsibilities [2]. Transmission of Mycobacterium tuberculosis is a recognized risk in health care facilities. It is most likely to occur from patients who have unrecognized pulmonary or laryngeal $\mathrm{TB}$, who are not on effective antiTB therapy and who are not placed in isolation [6]. The incidence of $M$. tuberculosis infection in health care workers varies widely, depending on such factors as practice location, patient population and local prevalence of TB. The baseline prevalence of past TB infection in health care workers has been reported to range from $1 \%$ to $28 \%$ [7]. In 1997, the Occupation Safety and Health Administration (OSHA) estimated that more than 5 million US workers were exposed to TB in the course of their work: in hospitals, homeless shelters, nursing homes and other work settings [8]. Unfortunately, prevalence rates of TB among health care workers in Saudi Arabia are not available, even though it is considered one of the most common chronic infectious diseases in the country.

The tuberculin skin test is the recommended instrument for TB screening of health care workers [5]. In several reports, from $4 \%$ to $79 \%$ of health care workers exposed to M. tuberculosis develop a positive tuberculin skin test [9]. Without known exposure, the yearly conversion rate of tuberculin for health care workers averages $0.1 \%-5.0 \%$ [7].

The Centers for Disease Control and Prevention (CDC) recommends that all health care workers should be screened with yearly tuberculin tests and the results should be interpreted without regard to previous BCG (bacille Calmette-Guérin) vaccine [5]. This recommendation represents a change from past practice, which suggested no skin testing after the receipt of BCG vaccine. Concomitantly, OSHA guidelines mandate the use of testing for TB in all new employees at risk for $M$. tuberculosis exposure who have not had a tuberculin skin test in the past year [10]. Positive tests indicate past TB exposure or infection and thus require further evaluation. Health care workers whose tuberculin tests have recently become positive should be evaluated for chemoprophylaxis after a chest radiograph is obtained to rule out active disease.

King Abdulaziz University Hospital (KAUH) has 400 beds and is a secondarytertiary medical centre in Jeddah. It covers referrals from a wide area of the western region of Saudi Arabia. Conducting tuberculin tests for health care workers at KAUH provided an opportunity to record the prevalence of positive tuberculin tests in order to better understand the risk of exposure to $\mathrm{TB}$, its spread and its role in health care occupations.

\section{Methods}

This study was performed in the staff health clinics of KAUH, Jeddah, Saudi Arabia. The outpatient and emergency room committee of KAU approved this work.

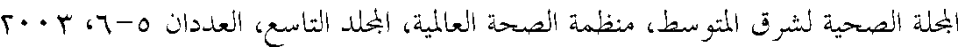


Participants in the present study were health care workers from all departments of KAUH. Between October 2000 and February 2001 they were invited to the staff health clinics for their tuberculin screening tests by letter sent through the heads of departments. Staff who responded were included (300 out of 400, 75\%). The medical records of the participants were reviewed by the clinic doctors and participants were interviewed about chronic medical illness and drug history. Participants were not suffering from any chronic illnesses (most of them are contract workers who can only obtain a work permit if they are medically fit) or taking regular medication (including steroids or immunosuppressants), and had no previous active TB infection. All participants had received BCG vaccination, since this is a compulsory vaccine in the country and all workers, both Saudi Arabian and non-Saudi Arabian, must provide a certificate of vaccination.

Screening was conducted using the Mantoux test (intracutaneous tuberculin units manufactured by Aventis Pasteur, France). Purified protein derivative supplies were provided by the pharmacy of KAUH. A nurse sterilized the forearm skin of the candidate with propylalcohol and, using a 26 gauge needle, introduced $0.1 \mathrm{~mL}$ of a commercially available purified protein derivative tuberculin intracutaneously (equivalent to 5 tuberculin units), raising a wheal 6-10 mm in diameter. The delayed skin test reactions were read by a physician after 48-72 hours, as convenient for the participants. The diameter of the erythematous induration was measured in $\mathrm{mm}$ and recorded. A positive tuberculin test was diagnosed if the wheal reaction was $10 \mathrm{~mm}$ or over. The conduct and interpretation of tuberculin skin tests were based on current guidelines of the CDC Committee on Latent Tuberculosis Infection $[5,11]$.
The data were entered into a personal computer. Frequency tables and determination of significant differences among variables were performed by the chi-squared test and Student $t$-test using SPSS, version 10.

\section{Results}

Of the 300 health care workers recruited, 2 did not attend at the appropriate time for the test reading, leaving 298 participants in the study. Their ages ranged between 21 and 59 years with a mean \pm standard deviation of $37.8 \pm 7.9$ years. Women were predominant, comprising $80.5 \%$ of the studied group. There were 60 participants from the laboratories (20.1\%), 26 from the neonatal intensive care unit (8.7\%), 24 from the paediatric medical ward (8.1\%) and 188 (63.1\%) from other departments.

The prevalence of a positive skin test (induration $\geq 10 \mathrm{~mm}$ ) in participants at KAUH was high, with 235 cases (78.9\% of the studied group). The most common sizes of skin test indurations were $20 \mathrm{~mm}$ (positive) for 47 participants (15.8\%), 10 $\mathrm{mm}$ (positive) for $46(15.4 \%)$ and $0 \mathrm{~mm}$ (negative) for 27 (9.1\%) (Figure 1). The mean values were $15.9 \pm 5.5 \mathrm{~mm}$ for positive reactions and $3.2 \pm 3.1 \mathrm{~mm}$ for negative reactions. Only a few participants, in particular those who had large tuberculin reactions, complained of local discomfort.

The non-Saudi Arabians were predominantly of Philippine (150 cases, 50.3\%) and Indian nationalities (36 cases, 12.1\%) (Table 1 ). The overall size of skin test reactions was significantly lower among Saudi Arabian participants than among non-Saudi Arabians (mean $8.9 \pm 7.1 \mathrm{~mm}$ versus $13.9 \pm 7.1 \mathrm{~mm})(t=4.1, P<0.001)$.

The distribution of positive skin test reactions among the different nationalities of

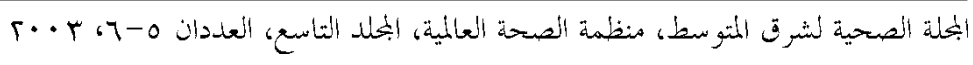




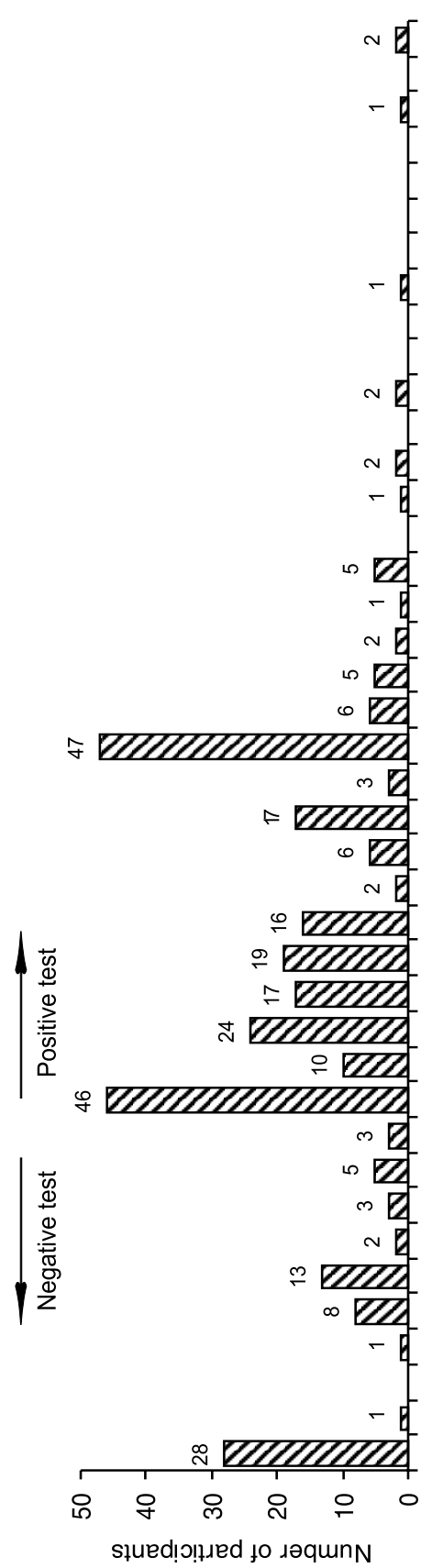

Figure 1 Distribution of positive and negative tuberculin skin test reactions for health care workers $(n=298)$ participants was assessed. The prevalence of positive reactions was highest for Nigerian $(87.5 \%)$ and Philippine participants (83.3\%). Among the Saudi Arabians, positive reactions were found in 24 cases of 40 (60.0\%), significantly fewer than among non-Saudi Arabian nationalities (81.8\%) $\left(\chi^{2}\right.$ $=9.9, P<0.01)$. Additionally, significantly fewer Saudi Arabians had positive reactions than non-Saudi Arabians (mean $13.6 \pm 4.5$ $\mathrm{mm}$ versus $16.2 \pm 5.5 \mathrm{~mm})(t=2.2, P<$ 0.05 ) (Table 2). The small number of participants for some of the nationality groups was not suitable for further analysis.

\section{Discussion}

The number of reported cases of TB is on the rise, concern about the disease has increased and more attention is being given to control measures. Therefore, this trial explored the prevalence of positive tuberculin tests among health care workers at KAUH.

Although the tuberculin skin test is the only available method for detecting $M$. tuberculosis infection, the test is neither $100 \%$ sensitive nor $100 \%$ specific [5]. In populations with a high prevalence of infection with non-tuberculous mycobacteria or vaccination with BCG, the specificity of the tuberculin test will be low. The positive predictive value of the tuberculin test is also variable. As the prevalence of TB infection in the population decreases, the positive predictive value of the tuberculin test also decreases. Appropriate interpretation of skin test results requires knowledge of: the antigen used (tuberculin); the immunological basis for the reaction to it; the technique of administering and reading the test; and the results of epidemiological and clinical experience with the test $[11,12]$.

The prevalence of positive tuberculin tests among the studied health care work-

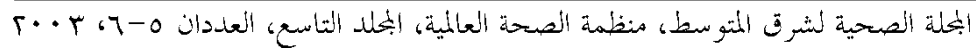




\begin{tabular}{|c|c|c|c|c|}
\hline \multirow[t]{2}{*}{ Nationality } & \multicolumn{2}{|c|}{ Total } & \multicolumn{2}{|c|}{ Test reaction size (mm) } \\
\hline & No. & $\%$ & Range & Mean $\pm s$ \\
\hline Saudi Arabian & 40 & 13.4 & $0-25$ & $8.9 \pm 7.1^{a}$ \\
\hline Non-Saudi Arabian & 258 & 86.6 & $0-40$ & $13.9 \pm 7.1$ \\
\hline Philippine & 150 & 50.3 & $0-40$ & $13.8 \pm 6.7$ \\
\hline Indian & 36 & 12.1 & $0-30$ & $13.8 \pm 7.2$ \\
\hline Pakistani & 31 & 10.4 & $0-40$ & $14.5 \pm 9.8$ \\
\hline Nigerian & 24 & 8.1 & $0-28$ & $15.3 \pm 6.6$ \\
\hline Other & 17 & 5.7 & $0-20$ & $13.3 \pm 5.4$ \\
\hline Total & 298 & 100.0 & $0-40$ & $13.3 \pm 7.3$ \\
\hline
\end{tabular}

${ }^{a} \mathrm{t}=4.1, \mathrm{P}<0.001$ Saudi versus non-Saudi participants. $\mathrm{s}=$ standard deviation.

ers at KAUH was high (79\%). In comparison to other studies, similar high prevalences of positive tuberculin tests (79\% and $70 \%)$ were found among medical service workers in Cote d'Ivoire and in a Mexican hospital $[13,14]$.

Interestingly, although positive tuberculin tests among health care workers holding Saudi Arabian nationality were elevated
(60\%), they were significantly lower in prevalence and reaction size than among non-Saudi Arabian nationalities. These differences could be attributed to variable socioeconomical factors and hygiene habits. However, the overall high prevalence of positive tuberculin tests among health care workers at KAUH may reflect the increased

Table 2 Distribution of positive tuberculin skin test reactions among the different nationalities of health care workers

\begin{tabular}{lrrrrr}
\hline Nationality & Total & \multicolumn{2}{c}{$\begin{array}{c}\text { Positive reaction } \\
\text { No. }\end{array}$} & \multicolumn{1}{c}{ No. } & \multicolumn{1}{c}{ Test reaction size $(\mathbf{m m})$} \\
\hline Saudi Arabian & 40 & 24 & $60.0^{\mathrm{a}}$ & $10-25$ & $13.6 \pm 4.5^{\mathrm{b}}$ \\
Nange & Mean $\pm \boldsymbol{s}$ \\
$\quad$ Philippine & 150 & 125 & 83.3 & $10-40$ & $16.2 \pm 5.5$ \\
$\quad$ Indian & 36 & 27 & 75.0 & $10-40$ & $15.9 \pm 5.2$ \\
Pakistani & 31 & 23 & 74.2 & $10-30$ & $16.1 \pm 5.9$ \\
$\quad$ Nigerian & 24 & 21 & 87.5 & $10-40$ & $18.8 \pm 7.1$ \\
$\quad$ Other & 17 & 15 & 88.2 & $10-20$ & $14.9 \pm 5.1$ \\
Total & 298 & 235 & 78.9 & $10-40$ & $15.9 \pm 5.5$ \\
\hline
\end{tabular}

${ }^{\mathrm{a}} \chi^{2}=9.9, \mathrm{P}<0.01$ Saudi versus non-Saudi participants.

$\mathrm{b}_{\mathrm{t}}=2.2, \mathrm{P}<0.05$ Saudi versus non-Saudi participants.

$\mathrm{s}=$ standard deviation. 
prevalence of TB cases not only in most of the developing countries but also in the Saudi Arabian community. In the general population of south-western Saudi Arabia, for example, positive tuberculin tests were reported for $4 \%$ of those aged 5-14 years and was even higher for those aged 45-64 years at up to $52 \%$ [15]. Positive tuberculin test rates have reached up to $20 \%$ among children from the Western provinces and Jeddah [16]. It is important to note that approximately $50 \%$ of the patients of KAUH are of non-Saudi Arabian nationality and that every year Jeddah receives over a million foreign pilgrims [16]. These factors and the lack of preventive programmes may be contributing issues in the increased risk of TB in Saudi Arabia.

Correlations between tuberculin test results and the different departments of these health care workers were investigated. Some authors found that the risk of positive tuberculin tests was higher for those who perform bronchoscopy [9]. Others found relatively lower conversion rates among emergency health care workers [17]. Unfortunately, such analysis was not feasible in the present study because of the small number of cases in some departments of KAUH and the frequent interchange of health care workers across the different departments.

In order to protect health care workers and to reduce the risk of TB exposure, the CDC Advisory Council for the Elimination of Tuberculosis recommends that health care facilities conduct a risk assessment and perform tuberculin skin testing at least annually and develop isolation procedures for potentially infectious TB patients in hospitals and in some outpatient settings $[4,5]$. Those whose tuberculin tests have recently become positive should be evaluated for chemo-prophylaxis, e.g. isoniazid, after a chest radiograph is obtained to ex- clude active disease. Prophylactic therapy should be considered because these patients are at high risk for TB. In recent times, multiple drug resistant strains of $M$. tuberculosis have become an increasing occupational concern among health care workers. If a health care worker exposed to a drug-resistant strain of $M$. tuberculosis has a skin test conversion, the prophylactic regimen may need to be modified or supplemented with drugs to which the infecting organism is susceptible.

Even when properly placed and interpreted, the tuberculin test may be associated with false positive or false negative results [5]. Causes of false positive tuberculin tests include error in administration, cross-reaction with non-tuberculous mycobacterial antigens, previous BCG vaccination and the booster phenomenon. The CDC recommends that, irrespective of their BCG vaccine status, health care workers should be screened with a yearly tuberculin test. Skin test reactions related to BCG vaccine typically decline in severity with time and the vaccine is not completely effective in preventing infection. If a tuberculin test response increases in size during yearly surveillance, a new infection is likely, rather than a reaction to previous BCG vaccination.

Some of the studied cases, particularly those with large tuberculin reactions, complained of discomfort, and refused such testing in the future. Generally, apart from local pain or discomfort, the tuberculin test is safe and valid even throughout pregnancy and has no known teratogenic effects [6]. There is no evidence that pregnancy affects the accuracy of tuberculin testing. The skin test is contraindicated if the worker has experienced a large necrotic reaction to past tuberculin testing.

OSHA guidelines recommend the use of 2-stage testing for TB in all new employees

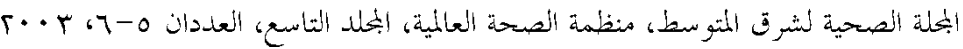


at risk for TB exposure who have not had a tuberculin test in the past year [10]. This 2stage procedure given 1 to 3 weeks after the first test is used to detect a possible 'boosting' effect that can occur when the first falsely negative tuberculin test leads to a 'recall' of the immune response. A positive second test constitutes a true positive, indicates past TB exposure or infection and thus requires further evaluation. Boosting can occur in $2 \%-10 \%$ of the general population $[18,19]$. The overall incidence of boosting in health care workers is not known. The CDC guidelines allow decisions about the use of 2-stage testing to be based on institutional data concerning the incidence of boosting in the facility. In this study, the 2-step test was not performed because of the high prevalence of positive tuberculin results.

Knowledge of the transmission of TB and infection control measures for TB among health care workers has been found to be incomplete [20]. Health administrators and infection control departments in hospitals are responsible for ensuring the implementation of an effective TB control programme. This requires education, risk assessment, early identification, isolation, complete treatment of infectious TB patients, effective engineering controls, an appropriate respiratory protection programme, counselling, screening and evaluation for health care workers.

A high prevalence of positive tuberculin tests was found among a subset of health care workers at KAUH. This might indicate a high risk for future acquisition of TB infection for the health care workers and subsequent spreading of disease. In order to enhance the protection of both health care workers and hospitalized patients, effective control and preventive measures for $\mathrm{TB}$, including yearly tuberculin testing of health care workers, should be considered.

\section{Acknowledgements}

The researchers would like to acknowledge Dr S. Matboli from the health staff clinics at King Abdulaziz University Hospital for her contribution in reading tuberculin tests. Additionally, the researchers acknowledge Dr W.A. Milaat from the Department of Community Medicine at King Abdulaziz University for his helpful support and peer review.

\section{References}

1. Rosenman KD, Hall N. Occupational risk factors for developing tuberculosis. American journal of industrial medicine, 1996, 30(2):148-54.

2. Swinker M. Occupational infections in health care workers: prevention and intervention. American family physician, 1997, 56(9):2291-300.

3. McCray $E$ et al. The epidemiology of tuberculosis in the United States. Clinics in chest medicine, 1997, 18:99-113.
4. Tuberculosis morbidity-United States, 1997. Morbidity and mortality weekly report, 1998, 47:253-6.

5. Screening for tuberculosis and tuberculosis infection in high risk populations. Recommendations of the Advisory Council for the Elimination of Tuberculosis. Morbidity and mortality weekly report, 1995, 44:18-34.

6. Guidelines for preventing the transmission of Mycobacterium tuberculosis in 
health care facilities, 1994. Centers for Disease Control and Prevention. Morbidity and mortality weekly report, 1994, 43(RR-13):1-132.

7. Bowden K, McDiarmid M. Occupationally acquired tuberculosis: what's known. Journal of occupational medicine, 1994, $36: 320-5$.

8. Occupational Safety and Health Administration. Occupational exposure to tuberculosis-proposed rule. Washington DC, US Department of Labor, 1977 (29 CFR Part 1910, docket no. H-371, RIN 1218-AB46:54160).

9. Sbarbaro JA. Tuberclosis: yesterday, today, and tomorrow [editorial]. Annals of internal medicine, 1995, 122:955-6.

10. Occupational Safety and Health Administration. Enforcement procedures and scheduling for occupational exposure to tuberculosis. Washington DC, US Department of Labor, Office of Health Compliance Assistance, 1996 (Directive no. CPL 2.106).

11. Jerant A, Bannon M, Ritteenhouse S. Identification and management of tuberculosis. American family physician, 2000, 61(9):2667-78.

12. American Thoracic Society, Medical Section of the American Lung Association. Diagnostic standards and classification of tuberculosis. American review of respiratory disease, 1990, 142(3):725-35.

13. Kassim $S$ et al. Tuberculosis skin testing to assess the occupational risk of $\mathrm{Myco}$ bacterium tuberculosis infection among health care workers in Abidjan, Cote d'Ivoire. International journal of tuberculosis and lung disease, 2000, 4(4):3216.

14. Gamboa MJ, Morales FI, Rosales PS. Prevalence of tuberculin reactivity among healthcare workers from a Mexican hospital. Infectious control and hospital epidemiology, 1994, 15(5):319-20.

15. Bener A. Prevalence of tuberculosis infection in ABHA and BAHA. European journal of epidemiology, 1990, 6(4): 376-81.

16. Al-Kassemi FA et al. Nationwide community survey of tuberculosis epidemiology in Saudi Arabia. Tubercle and lung disease, 1993, 74(4):254-60.

17. Prezant DJ et al. Tuberculosis skin test conversion rates in New York city emergency medical service health care workers. Annals of emergency medicine, 1998, 32(2):208-13.

18. Menzies $\mathrm{R}$ et al. The booster effect in two-step tuberculin testing among young adults in Montreal. Annals of internal medicine, 1994, 120:190-8.

19. Manusov EG, Bradshaw RD, Fogarty JP. Tuberculosis screening in medical students. Family medicine, 1996, 28:645-9.

20. Lai KK et al. Knowledge of the transmission of tuberculosis and infection control measures for tuberculosis among healthcare workers. Infectious control and hospital epidemiology, 1996, 17(3): 168-70.

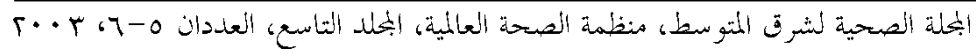

